\title{
DETECTION OF ROTATIONAL SPECTRAL VARIATION ON THE M-TYPE ASTEROID (16) PSYCHE
}

\author{
Juan A. Sanchez ${ }^{1,7}$, Vishnu Reddy ${ }^{2,7}$, Michael K. Shepard ${ }^{3}$, Cristina Thomas ${ }^{1,7}$, Edward A. Cloutis ${ }^{4}$, \\ Driss Takir ${ }^{5,7}$, Albert Conrad ${ }^{6}$, CAin KidDell ${ }^{4}$, and Daniel Applin ${ }^{4}$ \\ ${ }^{1}$ Planetary Science Institute, Tucson, AZ 85719, USA; jsanchez@psi.edu \\ ${ }^{2}$ Lunar and Planetary Laboratory, University of Arizona, Tucson, AZ 85721, USA \\ ${ }^{3}$ Bloomsburg University, Bloomsburg, PA 17815, USA \\ ${ }_{5}^{4}$ Department of Geography, University of Winnipeg, Winnipeg, Manitoba, Canada \\ ${ }^{5}$ Astrogeology Science Center, U.S. Geological Survey, Flagstaff, AZ 86001, USA \\ ${ }^{6}$ LBT Observatory, University of Arizona, Tucson, AZ 85721, USA \\ Received 2016 July 29; revised 2016 November 14; accepted 2016 November 15; published 2016 December 28
}

\begin{abstract}
The asteroid (16) Psyche is of scientific interest because it contains $\sim 1 \%$ of the total mass of the asteroid belt and is thought to be the remnant metallic core of a protoplanet. Radar observations have indicated the significant presence of metal on the surface with a small percentage of silicates. Prior ground-based observations showed rotational variations in the near-infrared (NIR) spectra and radar albedo of this asteroid. However, no comprehensive study that combines multi-wavelength data has been conducted so far. Here we present rotationally resolved NIR spectra $(0.7-2.5 \mu \mathrm{m})$ of (16) Psyche obtained with the NASA Infrared Telescope Facility. These data have been combined with shape models of the asteroid for each rotation phase. Spectral band parameters extracted from the NIR spectra show that the pyroxene band center varies from $\sim 0.92$ to $0.94 \mu \mathrm{m}$. Band center values were used to calculate the pyroxene chemistry of the asteroid, whose average value was found to be $\mathrm{Fs}_{30} \mathrm{En}_{65} \mathrm{Wo}_{5}$. Variations in the band depth (BD) were also observed, with values ranging from $1.0 \%$ to $1.5 \%$. Using a new laboratory spectral calibration method, we estimated an average orthopyroxene content of $6 \% \pm 1 \%$. The mass-deficit region of Psyche, which exhibits the highest radar albedo, also shows the highest value for the spectral slope and the minimum BD. The spectral characteristics of Psyche suggest that its parent body did not have the typical structure expected for a differentiated body or that the sequence of events that led to its current state was more complex than previously thought.
\end{abstract}

Key words: minor planets, asteroids: general - techniques: spectroscopic

\section{INTRODUCTION}

The asteroid (16) Psyche is often regarded as the archetype of M-type asteroids, which were originally defined by Zellner and Gradie (1976) and later included in the Tholen taxonomic system (Tholen 1984). They are characterized as having moderate albedos $(\sim 0.1-0.3)$ and featureless red-sloped spectra in the visible wavelength region $(0.3-1.1 \mu \mathrm{m})$. Among these objects, Psyche is the largest, with a diameter of $226 \pm 15 \mathrm{~km}$ (Shepard et al. 2017). It has a rotation period of $\sim 4.2 \mathrm{hr}$ (Kaasalainen et al. 2002) and a visual albedo of 0.12 (Tedesco et al. 2002). Early observations showed similarities between Psyche and iron meteorites (Chapman \& Salisbury 1973; Chapman \& Gaffey 1979), which led to the assumption that this asteroid is the remnant metallic core of a differentiated body that was catastrophically disrupted (e.g., Bell et al. 1989, p. 921). Numerous observations of Psyche using different techniques seem to support this theory. Radar observations have shown that Psyche has a radar albedo almost three times as high as the average value measured for S- and C-type asteroids (Ostro et al. 1985; Magri et al. 2007; Shepard et al. 2008, 2017), consistent with an object that is predominantly metallic. Kuzmanoski and Kovačević (2002) studied the perturbing effects caused by Psyche on the motion of 13206 Baer (1997 GC22) during a close encounter between

\footnotetext{
${ }^{7}$ Visiting Astronomer at the Infrared Telescope Facility, which is operated by the University of Hawaii under Cooperative Agreement no. NNX-08AE38A with the National Aeronautics and Space Administration, Science Mission Directorate, Planetary Astronomy Program.
}

these two asteroids. This yielded a density estimation of $6.98 \pm 0.58 \mathrm{~g} \mathrm{~cm}^{-3}$ for Psyche, a value that closely resembles that of iron meteorites $\left(7.5 \mathrm{~g} \mathrm{~cm}^{-3}\right)$. Similarly, estimations by Shepard et al. (2017) from radar observations led to a bulk density of $4.5 \pm 1.4 \mathrm{~g} \mathrm{~cm}^{-3}$ for this asteroid, consistent with an $\mathrm{Fe}-\mathrm{Ni}$ composition and a $40 \%$ macroporosity. Matter et al. (2013) estimated Psyche's thermal inertia from interferometric observations obtained with the VLT Interferometer, finding values of $133 \mathrm{~J} \mathrm{~m}^{-2} \mathrm{~s}^{-0.5} \mathrm{~K}^{-1}$ and $114 \mathrm{~J} \mathrm{~m}^{-2} \mathrm{~s}^{-0.5} \mathrm{~K}^{-1}$ (no roughness and low roughness, respectively). These high values were considered as evidence of a metal-rich surface for Psyche (Matter et al. 2013). In the NIR wavelength range $(\sim 0.7-2.5 \mu \mathrm{m})$ the spectrum of Psyche exhibits a red slope and a weak absorption band at $\sim 0.9 \mu \mathrm{m}$ (Clark et al. 2004; Hardersen et al. 2005; Ockert-Bell et al. 2010). These spectral characteristics are consistent with a surface metal component and a low-Fe pyroxene phase. Binzel et al. (1995) carried out rotationally resolved observations of Psyche in the visible wavelength range $(0.5-0.9 \mu \mathrm{m})$, finding no significant variations within a precision of $1 \%$. However, Ockert-Bell et al. (2008) reported variations in the spectral slope and the $0.9 \mu \mathrm{m}$ band depth (BD) from NIR spectra $(0.8-2.5 \mu \mathrm{m})$ of Psyche.

The physical characteristics and origin of Psyche make this asteroid an interesting case for study, as demonstrated not only by the extensive work carried out so far, but also by the prospect of an unmanned mission to this object (Elkins-Tanton et al. 2014, 2015). Here we present new rotationally resolved NIR spectra of (16) Psyche. In Section 2 we describe the observations and data reduction procedure. In Section 3 the 
Table 1

Observational Circumstances

\begin{tabular}{|c|c|c|c|c|c|c|c|}
\hline $\begin{array}{l}\text { Date } \\
\text { (UTC) }\end{array}$ & Mid UTC & $\begin{array}{c}\text { Lat. } \\
\text { (degree) }\end{array}$ & $\begin{array}{l}\text { Long. } \\
\text { (degree) }\end{array}$ & $\begin{array}{c}\text { Rot. Phase } \\
\text { (degree) }\end{array}$ & $\begin{array}{c}\alpha \\
\text { (degree) }\end{array}$ & $\begin{array}{c}\text { Mag. } \\
(V)\end{array}$ & Airmass \\
\hline 2015 Dec 08 & $10: 30: 38$ & -46.74 & 346 & 14 & 1.8 & 9.4 & 1.00 \\
\hline 2015 Dec 08 & 11:43:40 & -46.75 & 242 & 118 & 1.8 & 9.4 & 1.06 \\
\hline 2015 Dec 08 & $12: 47: 12$ & -46.76 & 151 & 209 & 1.8 & 9.4 & 1.20 \\
\hline 2015 Dec 09 & $14: 01: 48$ & -46.99 & 145 & 215 & 1.7 & 9.4 & 1.63 \\
\hline 2016 Feb 10 & $05: 10: 23$ & -52.19 & 70 & 290 & 19.5 & 10.8 & 1.02 \\
\hline 2016 Feb 10 & 05:30:41 & -52.19 & 41 & 319 & 19.5 & 10.8 & 1.01 \\
\hline 2016 Feb 10 & $06: 34: 30$ & -52.19 & 309 & 51 & 19.5 & 10.8 & 1.02 \\
\hline 2016 Feb 10 & $08: 05: 17$ & -52.18 & 180 & 180 & 19.5 & 10.8 & 1.17 \\
\hline
\end{tabular}

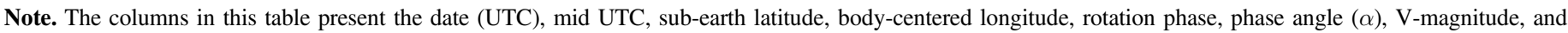
airmass. The rotation phases are based on the shape model and are calculated by subtracting the longitude from $360^{\circ}$.

NIR spectra are combined with shape models of the asteroid for each rotation phase. Band parameters are extracted from the spectra and used to determine the pyroxene chemistry and abundance across the surface of the asteroid. The effects of the phase angle, grain size, and space weathering on the spectra are also discussed. The global composition of Psyche and possible formation scenarios for this asteroid are discussed in Section 4. Finally, in Section 5 we summarize our main findings.

\section{OBSERVATIONS AND DATA REDUCTION}

NIR spectra ( $\sim 0.7-2.5 \mu \mathrm{m})$ of (16) Psyche were obtained on three nights between December 2015 and February 2016 using the SpeX instrument (Rayner et al. 2003) on the NASA Infrared Telescope Facility (IRTF) on Maunakea, Hawaii. The spectra were obtained using SpeX in its low-resolution $(R \sim 150)$ prism mode with a $0 . ! 8$ slit width. A G-type local extinction star was observed before and after the asteroid in order to correct for telluric features. Spectra of the solar analog star Hyades 64 (SAO 93936) were also acquired to correct for spectral slope variations that could arise due to the use of a non-solar extinction star. During the observations, the slit was oriented along the parallactic angle in order to reduce the effects of differential atmospheric refraction. The observational circumstances are presented in Table 1. Data reduction was performed using the IDL-based software Spextool (Cushing et al. 2004). A detailed description of the steps followed in the data reduction process can be found in Sanchez et al. (2013, 2015).

\section{RESULTS}

\subsection{Spectral Band Parameters Versus Rotation Phase}

NIR spectra of (16) Psyche corresponding to eight different body-centered longitudes are shown in Figure 1. This figure also includes views of the shape model of Psyche for each rotation phase. Shepard et al. (2017) used 18 Arecibo delayDoppler radar observations and six new adaptive optics images from Keck and Magellan to generate a three-dimensional shape model of Psyche using the SHAPE software package (Magri et al. 2007). The shape model incorporates the spin-state and ephemeris so that for any time in the past or future, a plane-ofsky view can be generated to correlate with spectra or other telescopically derived data. For the shape model, we adopted a rotation period $P=4.195948 \pm 0.000001 \mathrm{hr}$ and a spin pole of $(\lambda, \beta)\left(34^{\circ},-7^{\circ}\right) \pm 5^{\circ}$. For each of our IRTF runs, we generated plane-of-sky views using the mid-point time of our observations.

As can be seen in Figure 1, the spectra look relatively homogeneous across the surface of the asteroid, with all of them showing a weak absorption band at $\sim 0.9 \mu \mathrm{m}$. This feature has been detected by previous studies and has been attributed to the presence of low-Fe orthopyroxene (e.g., Clark et al. 2004; Hardersen et al. 2005; Ockert-Bell et al. 2010; Neeley et al. 2014). For each spectrum, we measured the spectral band parameters, band center, BD, and spectral slope using a Python code and following the procedure described in Cloutis et al. (1986). The band center was calculated by dividing out the linear continuum and fitting a polynomial over the bottom third of the band. The BD was measured as in Clark and Roush (1984) and is given as a percentage depth. The spectral slope is defined as the slope of a straight line fitted from 0.76 to $1.2 \mu \mathrm{m}$. Each band parameter was measured ten times by using thirdand fourth-order polynomial fits and sampling different ranges of points within the corresponding intervals. The reported values correspond to the average of these measurements. Uncertainties are given by the standard deviation of the mean calculated from the ten measurements of each parameter. Table 2 lists the band center, BD, and spectral slope values measured for each spectrum.

From our measurements we found that the band center varies from $\sim 0.92$ to $0.94 \mu \mathrm{m}$, with an average value of $0.932 \pm 0.006 \mu \mathrm{m}$, which is consistent with the values reported by previous studies (e.g., Hardersen et al. 2005; Fornasier et al. 2010; Ockert-Bell et al. 2010). Variations of the BD with the rotation phase were also observed, with values ranging from $1.0 \%$ to $1.5 \%$ and an average value of $1.3 \% \pm 0.1 \%$. Similarly, variations of the spectral slope with the rotation phase were found, with minimum and maximum values of 0.25 and 0.35 $\mu \mathrm{m}^{-1}$, respectively, and an average slope of $0.30 \pm 0.01 \mu \mathrm{m}^{-1}$. In Figures 2 and 3 we plot the BD and spectral slope versus the rotation phase, along with radar albedo measurements of Psyche from Shepard et al. (2008) and Shepard et al. (2017). A possible anti-correlation between the $\mathrm{BD}$ and radar albedo (i.e., increasing BD with decreasing radar albedo) can be seen.

Shepard et al. (2017) determined that Psyche has an ellipsoidal shape; however, they noticed a deficit of mass at longitudes between $0^{\circ}$ and $90^{\circ}$ at the equator. This mass-deficit region, which exhibits the highest radar albedo, shows the highest value for the spectral slope and the minimum BD, while the antipode of this region (longitudes $\sim 180^{\circ}-230^{\circ}$ ), where the radar albedo reaches its lowest value, shows the maximum BD and less steep spectral slopes. 

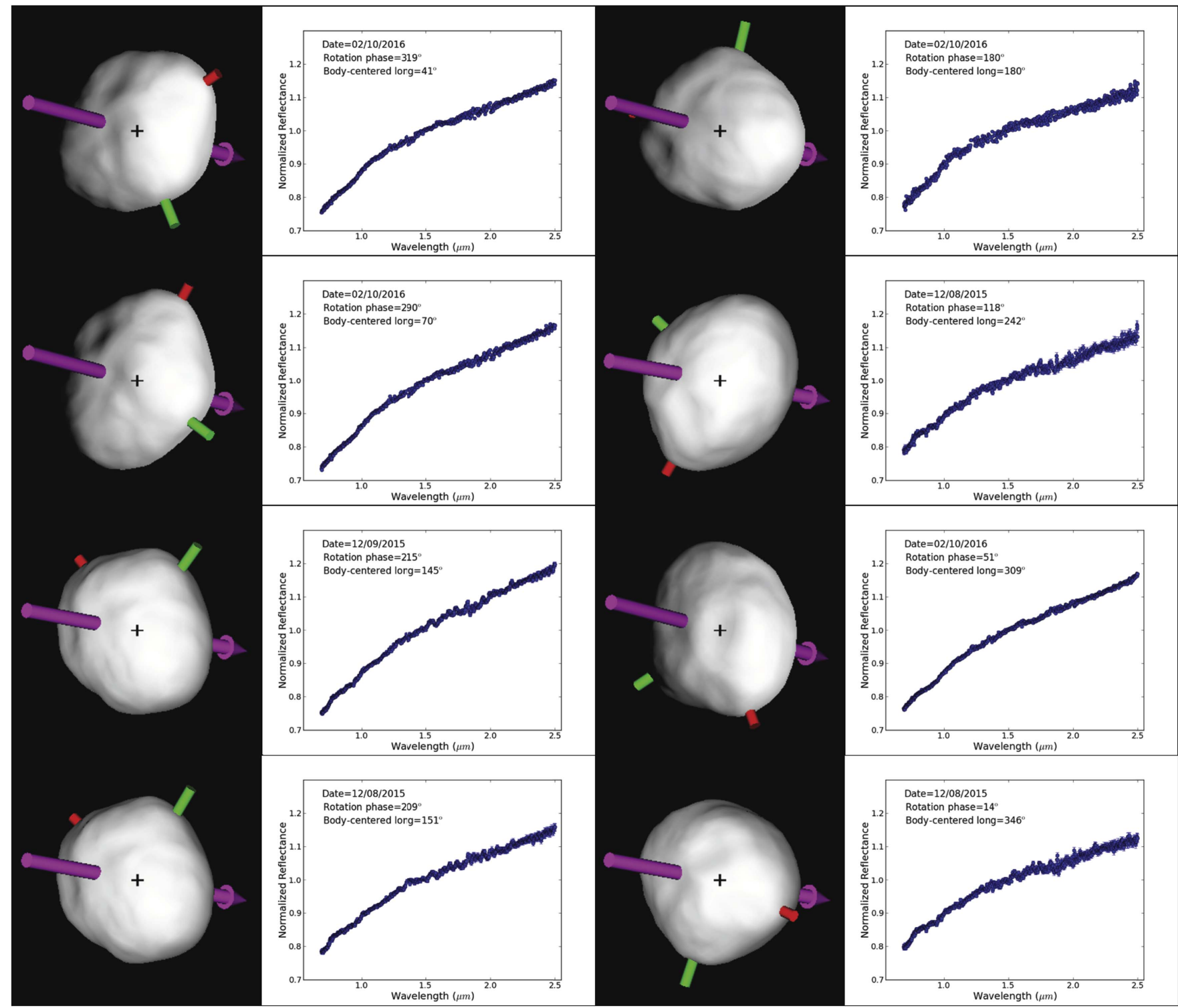

Figure 1. NIR spectra and shape models of (16) Psyche corresponding to eight different rotation phases. All spectra are normalized to unity at $1.5 \mu$ m. The axes shown on the shape models correspond to longitude $0^{\circ}$ (red) and longitude $90^{\circ}$ (green).

\subsection{Compositional Analysis}

\subsubsection{Rotational Variations in the Pyroxene Chemistry}

The variations observed in the band center could be attributed to differences in the pyroxene chemistry across the surface of the asteroid. Previous studies (e.g., Hardersen et al. 2005, 2011) calculated the pyroxene chemistry of only those M-types whose spectra exhibit both pyroxene bands (i.e., using the Band I and Band II centers), and since Band II, which is centered at $\sim 1.9-2 \mu \mathrm{m}$, is not present in the spectrum of Psyche, the pyroxene chemistry of this asteroid was never calculated. The pyroxene chemistry is given by the molar content of ferrosilite (Fs) and wollastonite (Wo) and can be calculated using Gaffey et al. (2002) or Burbine et al. (2007) calibrations. The equations from Gaffey et al. (2002) require both band centers; however, those derived by Burbine et al. (2007) can be used even if only one pyroxene band is present. Thus, the equations from Burbine et al. (2007) were used along with the measured Band I center. These equations were derived from the analysis of howardites, eucrites, and diogenites (HEDs) and therefore provide only a rough estimation. We determined that the pyroxene chemistry for Psyche ranges from $\mathrm{Fs}_{26.7 \pm 3.3} \mathrm{Wo}_{3.5 \pm 1.1}$ to $\mathrm{Fs}_{48.2 \pm 3.3} \mathrm{Wo}_{11.8 \pm 1.1}$, given an average of $\mathrm{Fs}_{40} \mathrm{En}_{51} \mathrm{Wo}_{9}$. The calculated values for each rotation phase are included in Table 2. This broad range of values must be taken with caution, since it is not clear whether they are the result of the scattering of the data or actual surface heterogeneities. Furthermore, the presence of an additional mafic silicate phase (e.g., olivine or high-Ca pyroxene) would shift the band center to longer wavelengths, without necessarily introducing a Band II, thereby causing an overestimation of the Fs content.

Comparing these results with those obtained by Hardersen et al. (2011), who studied a large sample of M-type asteroids, we notice that the average surface pyroxene chemistry of Psyche is very similar to that calculated for asteroids (338) Budrosa $\left(\mathrm{Fs}_{39} \mathrm{En}_{53} \mathrm{Wo}_{8}\right)$ and (497) Iva $\left(\mathrm{Fs}_{41} \mathrm{En}_{50} \mathrm{Wo}_{9}\right)$. However, in contrast to those of Psyche, the spectra of these two asteroids exhibit both pyroxene bands. According to Hardersen et al. (2011), the position of the band centers of Budrosa and 
Table 2

Spectral Band Parameters and Composition

\begin{tabular}{lccccrr}
\hline \hline $\begin{array}{l}\text { Lat. } \\
(\text { degree })\end{array}$ & $\begin{array}{c}\text { Long. } \\
(\text { degree })\end{array}$ & $\begin{array}{c}\text { BC } \\
(\mu \mathrm{m})\end{array}$ & $\begin{array}{c}\mathrm{BD} \\
(\%)\end{array}$ & $\begin{array}{c}\text { Slope } \\
\left(\mu \mathrm{m}^{-1}\right)\end{array}$ & $\begin{array}{c}\text { Fs } \\
(\mathrm{mol} \%)\end{array}$ & $\begin{array}{c}\text { Wo } \\
(\mathrm{mol} \%)\end{array}$ \\
\hline-46.74 & 346 & $0.940 \pm 0.010$ & $1.3 \pm 0.1$ & $0.25 \pm 0.01$ & $48.2 \pm 3.3$ & $11.8 \pm 1.1$ \\
-46.75 & 242 & $0.940 \pm 0.005$ & $1.4 \pm 0.1$ & $0.26 \pm 0.01$ & $48.2 \pm 3.3$ & $11.8 \pm 1.1$ \\
-46.76 & 151 & $0.935 \pm 0.010$ & $1.0 \pm 0.1$ & $0.27 \pm 0.01$ & $43.1 \pm 3.3$ & $9.8 \pm 1.1$ \\
-46.99 & 145 & $0.940 \pm 0.003$ & $1.2 \pm 0.1$ & $0.31 \pm 0.01$ & $48.2 \pm 3.3$ & $11.8 \pm 1.1$ \\
-52.19 & 70 & $0.935 \pm 0.004$ & $1.3 \pm 0.1$ & $0.35 \pm 0.01$ & $43.1 \pm 3.3$ & $9.8 \pm 1.1$ \\
-52.19 & 41 & $0.919 \pm 0.006$ & $1.0 \pm 0.1$ & $0.34 \pm 0.01$ & $26.7 \pm 3.3$ & $3.5 \pm 1.1$ \\
-52.19 & 309 & $0.919 \pm 0.003$ & $1.3 \pm 0.1$ & $0.31 \pm 0.01$ & $26.7 \pm 3.3$ & $3.5 \pm 1.1$ \\
-52.18 & 180 & $0.928 \pm 0.006$ & $1.5 \pm 0.1$ & $0.31 \pm 0.01$ & $35.9 \pm 3.3$ & $7.1 \pm 1.1$ \\
\hline
\end{tabular}

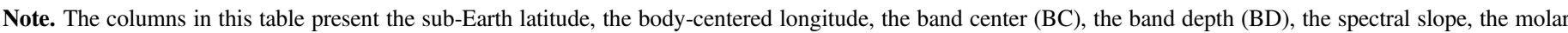

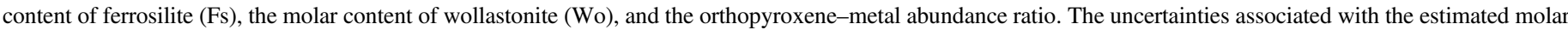

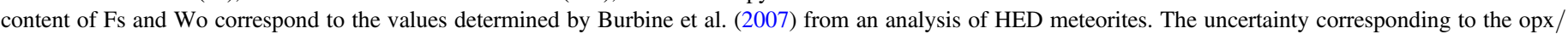
$(\mathrm{opx}+$ metal) ratio is given by the root mean square error between the opx $/(\mathrm{opx}+$ metal) ratio determined using Equation (1) and the real values.

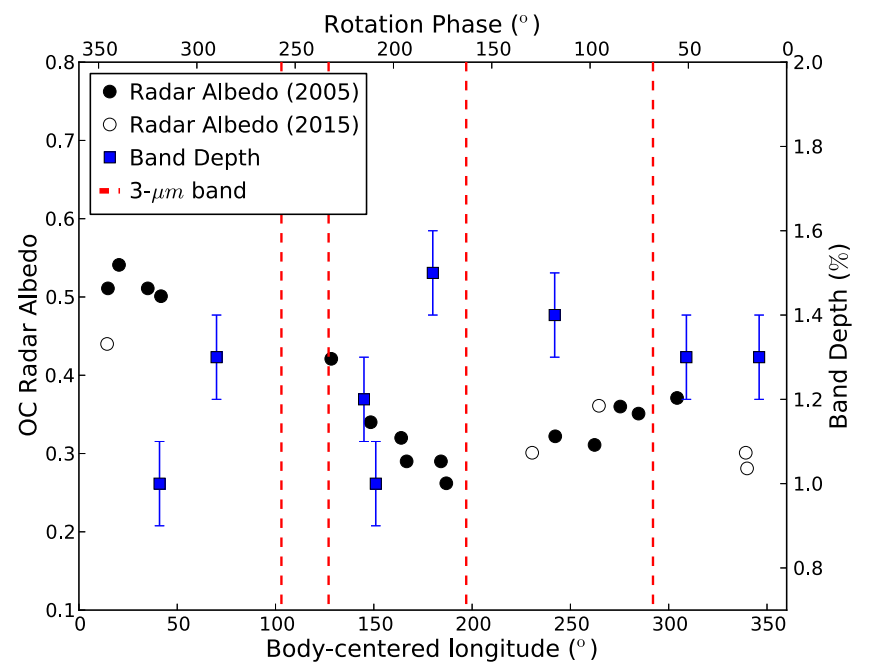

Figure 2. Band depth vs. body-centered longitude/rotation phase for (16) Psyche. Radar albedo measurements of Psyche from Shepard et al. (2008, 2017) are also shown. The mean OC radar albedo for Psyche is $\hat{\sigma}_{o c}=0.37 \pm 0.09$ (Shepard et al. 2017). The location of regions where the $3 \mu \mathrm{m}$ band has been detected by Takir et al. (2016) is depicted as red dashed lines.

Iva in the Band I versus Band II plot, "slightly above the pyroxene trend," could be attributed to the presence of either an olivine or a high-Ca pyroxene phase. The presence of these minerals could then lead to an overestimation of the Fs abundance. Thus, it is possible that there are no regions on Psyche that are more ferrous than others, but its regions contain residues of other minerals, like high-Ca pyroxene. Considering this, the lowest calculated values are likely more representative of the pyroxene chemistry of Psyche. Therefore, if we recalculate the average considering only the lowest values of Fs and Wo, corresponding to longitudes of $41^{\circ}, 180^{\circ}$, and $309^{\circ}$, we obtain $\mathrm{Fs}_{30} \mathrm{En}_{65} \mathrm{Wo}_{5}$. This value is within the range found for pyroxene megacrysts and the mafic melt matrix of mesosiderite meteorites (e.g., Boesenberg et al. 1997). Figure 4 shows the molar content of Fs as a function of body-centered longitude for Psyche. The lowest value of Fs is found for bodycentered longitudes of $41^{\circ}$ and $309^{\circ}$, with the former corresponding to a possible excavated region, perhaps similar to Vesta's Rheasilvia basin (Shepard et al. 2017), and the latter corresponding to the location of a crater-like depression $85 \pm 20 \mathrm{~km}$ in diameter found by Shepard et al. (2017). We

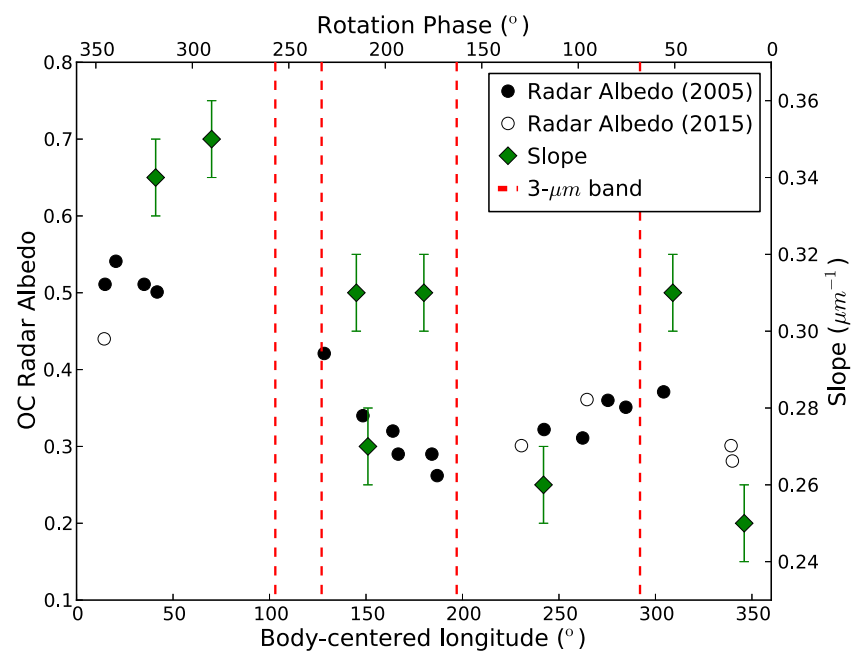

Figure 3. Spectral slope vs. body-centered longitude/rotation phase for (16) Psyche. Radar albedo measurements of Psyche from Shepard et al. (2008, 2017) are also shown. The mean OC radar albedo for Psyche is $\hat{\sigma}_{o c}=0.37 \pm 0.09$ (Shepard et al. 2017). The location of regions where the $3 \mu \mathrm{m}$ band has been detected by Takir et al. (2016) is depicted as red dashed lines.

notice that out of eight, five rotation phases exhibit high Fs content. If these high values are indeed the result of an overestimation caused by a secondary or accessory mineral, then this mineral is likely present across the surface of the asteroid, with only a few zones (possible excavated regions) with a minor content of it. A schematic map of Psyche is shown in Figure 5. All the spectral observations were obtained at a sub-Earth latitude of $\sim 50^{\circ} \mathrm{S}$. Low-Fe and moderate-Fe pyroxene regions are depicted in grayscale.

\subsubsection{Rotational Variations in Metal Abundance}

Variations in the BD and spectral slope could be the result of differences in mineral abundance. In order to explore this possibility, we calculate the fraction of pyroxene and metal present as a function of rotation phase. We choose these two minerals because they are the ones that better fit the spectral characteristics of Psyche. To this end, we have developed a new laboratory spectral calibration method using data from Cloutis et al. (2009), who measured the reflectance spectra of intimate mixtures composed of orthopyroxene $\left(\mathrm{Fs}_{12.8}\right)$ and metal from the Odessa octahedrite iron meteorite (grain size 


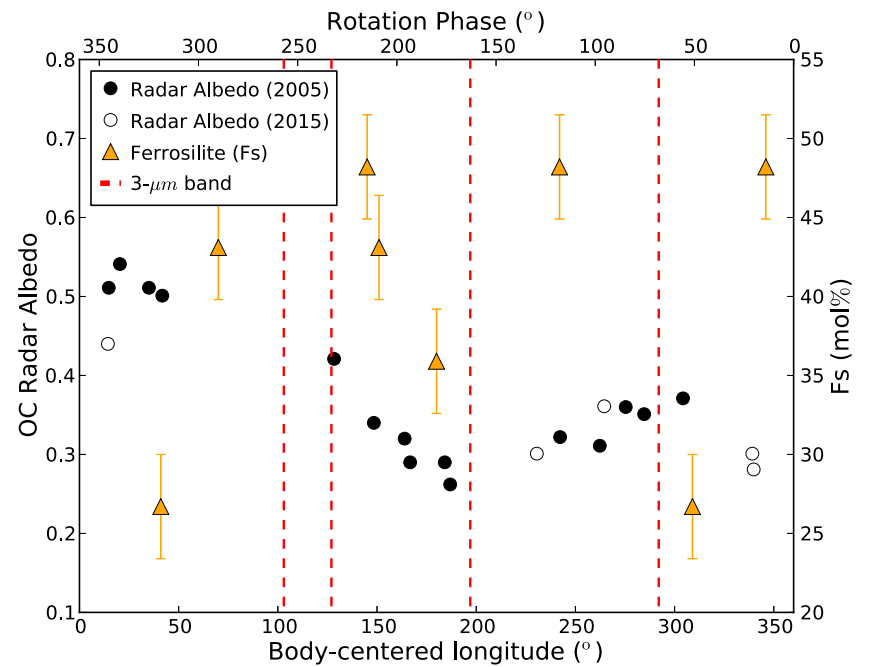

Figure 4. Molar content of ferrosilite (Fs) vs. body-centered longitude/rotation phase for (16) Psyche. Radar albedo measurements of Psyche from Shepard et al. $(2008,2017)$ are also shown. The mean OC radar albedo for Psyche is $\hat{\sigma}_{o c}=0.37 \pm 0.09$ (Shepard et al. 2017). The location of regions where the $3 \mu \mathrm{m}$ band has been detected by Takir et al. (2016) is depicted as red dashed lines.

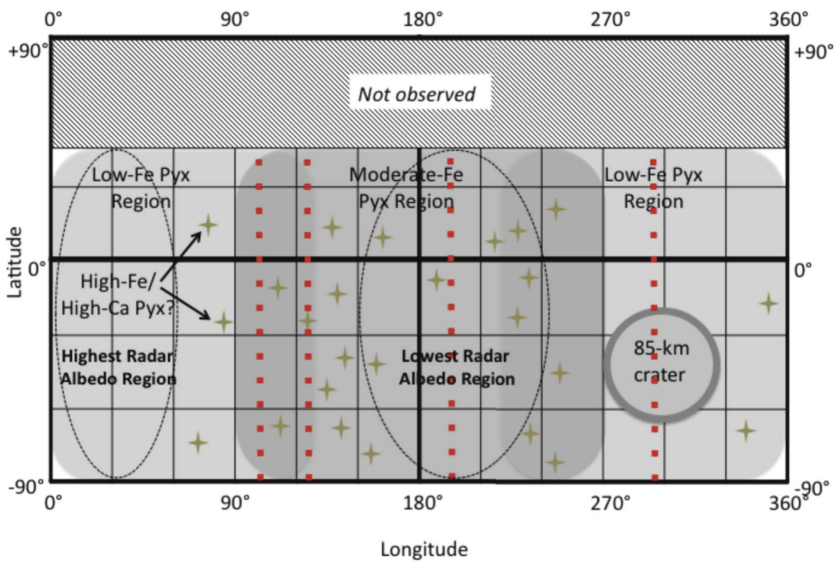

Figure 5. Schematic map of (16) Psyche. Low-Fe and moderate-Fe pyroxene (Pyx) regions are depicted in grayscale. The highest and lowest radar albedo regions from Shepard et al. (2017) are indicated as ovals. Star symbols are used to indicate that high-Fe pyroxene or a secondary phase (e.g., high-Ca pyroxene) might be present across the surface of the asteroid. A crater-like depression $85 \pm 20 \mathrm{~km}$ in diameter at a body-centered longitude of $300^{\circ}$ found by Shepard et al. (2017) is shown. The location of regions where the $3 \mu \mathrm{m}$ band has been detected by Takir et al. (2016) is depicted as red dashed lines.

$<45 \mu \mathrm{m}$ in both cases). The reflectance spectra of the samples correspond to intimate mixtures where the orthopyroxene abundance was increased in $10 \mathrm{wt} . \%$ intervals.

For each spectrum, we measured the band I depth in the same way as we did for the asteroid spectra. Figure 6 shows the orthopyroxene-metal abundance ratio $(\mathrm{opx} /(\mathrm{opx}+$ metal)) versus $\mathrm{BD}$ for the intimate mixtures. As noted by Cloutis et al. (2009), an increase in the metal content will cause the BD to decrease and the spectral slope to increase. We found that the correlation between opx/(opx+metal) and $\mathrm{BD}$ can be described by the following second-order polynomial fit:

$$
\begin{aligned}
\mathrm{opx} /(\mathrm{opx}+\text { metal })= & -0.000274 \times \mathrm{BD}^{2} \\
& +0.033 \times \mathrm{BD}+0.014
\end{aligned}
$$

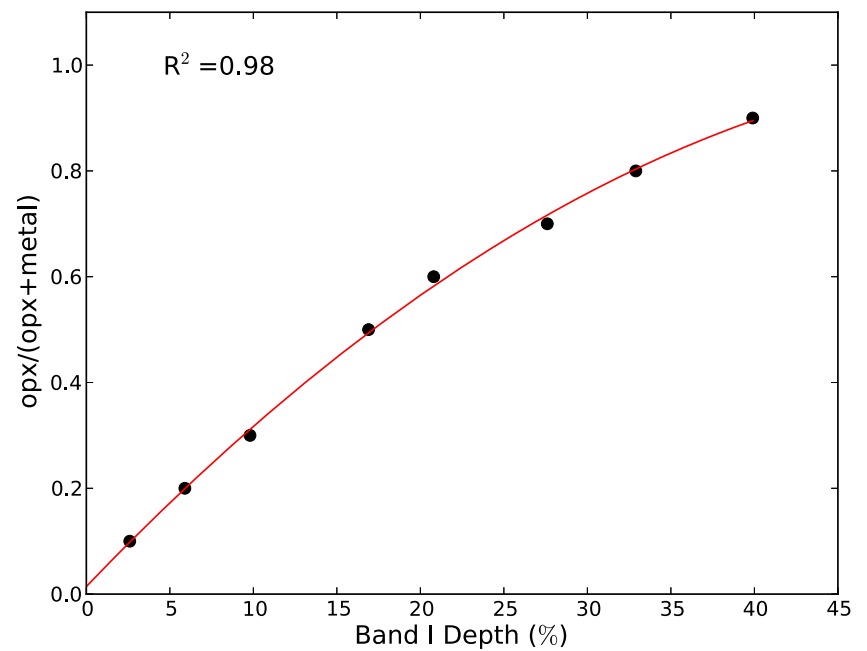

Figure 6. opx $/($ opx + metal $)$ ratios vs. BD for intimate mixtures composed of orthopyroxene and metal from the Odessa octahedrite iron meteorite. The red line represents a second-order polynomial fitted to the data. The coefficient of determination $\left(R^{2}\right)$ is given. The root mean square error between the opx/(opx + metal) ratio determined using Equation (1) and the real values is 0.01 .
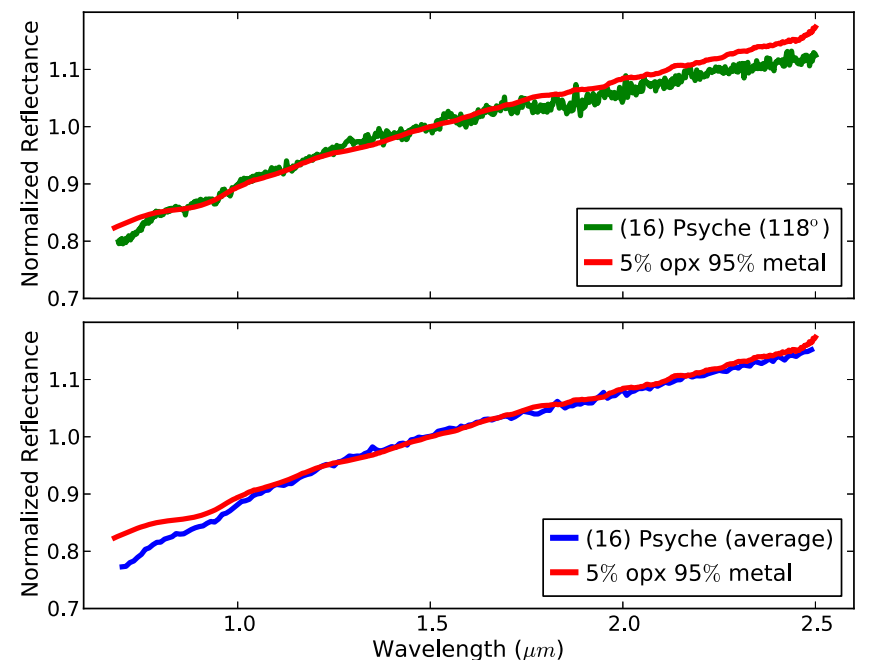

Figure 7. Comparison of the spectrum of an intimate mixture of $5 \mathrm{wt} \%$ orthopyroxene and $95 \mathrm{wt} \%$ metal from Cloutis et al. (2009) to the spectrum of Psyche corresponding to rotation phase $118^{\circ}$ (top) and to the average spectrum of Psyche (bottom). All spectra are normalized to unity at $1.5 \mu \mathrm{m}$.

where the coefficient of determination $\left(R^{2}\right)$ is 0.98 and the root mean square error between the opx $/(\mathrm{opx}+$ metal $)$ ratio determined using Equation (1) and the real values is 0.01 . Using this equation along with the BDs measured from each spectrum of Psyche, we determined the pyroxene abundance. These values are included in Table 2 . We found that the maximum variation of pyroxene content with the rotation phase is $1 \%$, with an average opx $/(\mathrm{opx}+$ metal) ratio of $6 \% \pm 1 \%$. The lowest opx $/($ opx + metal $)$ ratio corresponds to longitudes of $41^{\circ}, 145^{\circ}$, and $151^{\circ}$. These results indicate that a small difference in metal content could explain the variations of the $\mathrm{BD}$ and spectral slope with the rotation phase. However, since this difference is on the order of the uncertainty associated with the method we are using, we cannot be completely certain.

For comparison, in Figure 7 we show the spectrum of an intimate mixture of $5 \mathrm{wt} . \%$ orthopyroxene and $95 \mathrm{wt} \%$ metal 
from Cloutis et al. (2009). The overall shape of the spectrum of this intimate mixture is very similar to that of Psyche spectra, although some individual spectra fit better than others. In this example, we can see how the spectrum corresponding to rotation phase $118^{\circ}$ (longitude $242^{\circ}$ ) is more similar to that of the intimate mixture than the average spectrum of Psyche, whose spectral slope is slightly different. Since we have normalized the spectra to unity at $1.5 \mu \mathrm{m}$, the difference in spectral slope is seen as a drop in reflectance shortward of $1.2 \mu \mathrm{m}$.

\subsection{Effects of Phase Angle, Grain Size, and Space Weathering}

Variations in BD and spectral slope could be attributed to factors other than mineral abundance, including differences in phase angle, grain size, and space weathering. As can be seen in Table 1, observations of Psyche were obtained at two very different phase angles, 1.8 and $19^{\circ} .5$. An increase in phase angle can produce changes in the strength of the absorption band and increase the spectral slope (Sanchez et al. 2012) and could therefore explain the variations of these two parameters between different observing runs. However, this cannot explain the variations seen within each observing run. This opens the possibility for variations of grain size and space weathering with the rotation phase.

An increase in grain size will typically produce an increase in $\mathrm{BD}$, albedo variations, and a decrease in spectral slope (e.g., Cloutis et al. 2009, 2010, 2015). This alternative explanation would be compatible with the albedo variations across the surface of Psyche reported by previous studies (e.g., Kaasalainen et al. 2002). Furthermore, a change in surface texture is considered among the possible causes for the variations in radar albedo detected on Psyche (Shepard et al. 2008).

In the case of lunar-style space weathering, the effects on the spectra can be seen as an increase in spectral slope and a suppression of the absorption bands (e.g., Pieters et al. 2000; Hapke 2001; Brunetto et al. 2006; Gaffey 2010). Thus, rotation phases whose spectra exhibit less steep slopes and deeper absorption bands could represent regions on the surface where fresh material has been exposed. In addition, there is new evidence that suggests the presence of $\mathrm{H}_{2} \mathrm{O} / \mathrm{OH}$-bearing minerals on the surface of Psyche. In a recent study, Takir et al. (2016) detected a $3 \mu \mathrm{m}$ band in the mid-IR spectra $(1.9-4.1 \mu \mathrm{m})$ of Psyche. The locations where this band was detected are depicted in Figures 2-5 (red dashed lines). This $3 \mu \mathrm{m}$ band could be related to the presence of dark exogenic material (e.g., carbonaceous chondrites), like the one found on the surface of Vesta (e.g., Reddy et al. 2012). If this is the case, then the presence of the dark material could have also contributed to the variations seen in the spectral slope and BD.

\section{DISCUSSION}

The conditions that led to the formation of Psyche remain a mystery. As stated earlier, one of the most accepted theories is that this object is the exposed metallic core of an $\sim 500 \mathrm{~km}$ diameter differentiated body. The structure of a differentiated body, which has been molten and density-segregated, consists of an $\mathrm{FeNi}-\mathrm{FeS}$ core, an olivine-rich mantle, and a pyroxenefeldspar (basaltic) crust (e.g., Bell et al. 1989; Gaffey et al. 1993). Our analysis showed a relatively homogeneous metal content across the surface of Psyche, with only a small fraction
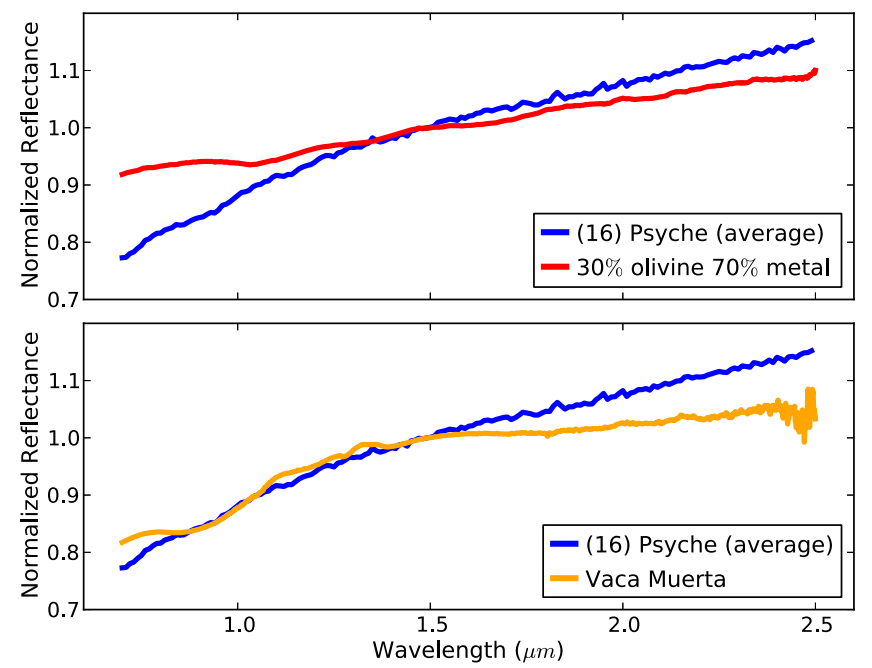

Figure 8. Top: NIR spectrum of an intimate mixture of $30 \mathrm{wt} . \%$ olivine and 70 wt.\% metal from Cloutis et al. (2015) compared to the average spectrum of Psyche. Bottom: NIR spectrum of the mesosiderite Vaca Muerta obtained at the University of Winnipeg HOSERLab compared to the average spectrum of Psyche. Differences between the two spectra at wavelengths $>1.5 \mu \mathrm{m}$ could be attributed to different grain sizes and metal abundance. Space weathering and/ or differences in metal grain size likely account for the spectral mismatch below $\sim 0.85 \mu \mathrm{m}$. All spectra are normalized to unity at $1.5 \mu \mathrm{m}$.

( $\sim 6 \%)$ of silicates. The silicate component, which is dominated by orthopyroxene, seems to be intimately mixed with the metal, as indicated by the presence in all of the spectra of a weak pyroxene absorption band. The possible presence of a secondary phase, possibly high-Ca pyroxene, across the surface suggests that this mineral was originally derived from the parent body. An interesting aspect about Psyche is the apparent lack or low content of olivine on the surface, as revealed by our observations and previous studies. Numerical simulations have shown that hit-and-run collisions can strip the silicate crust and mantle from differentiated bodies (e.g., Asphaug et al. 2006; Asphaug \& Reufer 2014). However, it is unlikely that this event would produce a pure metallic core, without any residual olivine mantle. Subsequent collisions and micrometeorite impacts would shatter the residual silicate fragments, producing a regolith layer mainly composed of metal and a small fraction of olivine. Figure 8 (top panel) shows an example of the NIR spectrum of an intimate mixture of $30 \mathrm{wt} . \%$ olivine and $70 \mathrm{wt}$. $\%$ metal from Cloutis et al. (2015). This spectrum exhibits a weak feature centered at $\sim 1.05 \mu \mathrm{m}$, very different from Psyche spectra, whose band center varies from $\sim 0.92$ to $0.94 \mu \mathrm{m}$. This suggests that Psyche's parent body did not have the typical structure expected for a differentiated body with an oxidized precursor or that the sequence of events that led to Psyche's current state was more complex than expected.

For planetesimals with different chondritic compositions that underwent melting and differentiation, the first crystallizing mineral in almost all cases is olivine, and therefore, this is the most likely silicate remnant to be present on the surface of Psyche (Elkins-Tanton et al. 2013). However, if the planetesimal had a silica-rich bulk composition (more reduced) and the pressure of the interior was sufficiently low, then the first crystallizing mineral would have been pyroxene (ElkinsTanton et al. 2013). If this was the case for Psyche's parent body, then this could explain the apparent lack of olivine on the surface of this asteroid. 
Another possible explanation could involve a formation scenario similar to the one proposed for mesosiderites' parent body. Mesosiderites are stony-iron meteorites composed of approximately equal amounts of $\mathrm{Fe}$, Ni-metal, and silicates plus troilite (Weisberg et al. 2006, p. 19). The silicate component is similar in composition to HED meteorites, containing olivine, pyroxenes, and plagioclase (Mittlefehldt et al. 1998, pp. 4-1-4495). One of the interesting features of these meteorites is that crustal and core materials are abundant, while olivine from the mantle is rare. Figure 8 (bottom panel) shows the NIR spectrum of a slab of the mesosiderite Vaca Muerta. As can be seen in this figure, the overall shape of the spectrum is similar to that of Psyche, with a pyroxene absorption band centered at $\sim 0.91 \mu \mathrm{m}$. The differences in overall slope beyond $\sim 1.5 \mu \mathrm{m}$ can be attributed to differences in metal grain size: Psyche likely has a powdered regolith, which would lead to a more red-sloped spectrum compared to our mesosiderite spectra, which were measured as points on a roughened slab. For metal +mafic silicates, slab spectra are less red-sloped than powders. For mesosiderite slab spectra, increasing metal content (as determined visually where spot spectra were acquired) results in more red-sloped spectra and shallower pyroxene absorption BDs, as expected. Space weathering would have the greatest effect at the lowest wavelengths, causing a reddening of the spectra slope. This is evident in the Psyche spectra versus the laboratory spectra of the mineral mixtures and mesosiderite slab spectra.

Numerical experiments performed by Scott et al. (2001) showed that mesosiderites could have been formed by the disruption and reassembly of a Vesta-like asteroid. In this scenario, the target, a differentiated asteroid with a molten core, is disrupted by a smaller projectile. According to Scott et al. (2001), after the disruption event most of the molten metal would have crystallized around the small, cool crustal fragments, as they were more efficient at trapping molten metal than the hotter and larger fragments from the mantle. As a result, olivine-rich mantle material would have been preferentially excluded from the metal-rich regions of the reaccreted body. The lower survival rate of the olivine fragments could have also contributed to reducing the olivine content in the reaccreted body, which would consist of a mixture of metal, pyroxenite, basalt, and dunite fragments. After the new reaccreted body was formed, subsequent collisions must have taken place, but this time with the reaccreted body acting as the impactor. The reason is that in a hit-and-run collision the impactor is more easily disrupted than the target it strikes, losing most of the outer layers in the process with no subsequent accretion (Asphaug et al. 2006; Asphaug \& Reufer 2014). These hit-and-run collisions would result in an exposed metallic core, probably retaining only a small fraction of silicates composed of intimately mixed materials from all depths. We speculate that this sequence of events could also explain the spectral characteristics seen on Psyche.

\section{SUMMARY}

We have obtained rotationally resolved NIR spectra of the asteroid (16) Psyche. Band parameters were measured from each spectrum corresponding to different rotation phases. We found variations of the band center $(0.92-0.94 \mu \mathrm{m})$, BD $(1.0 \%-1.5 \%)$ and spectral slope $\left(0.25-0.35 \mu \mathrm{m}^{-1}\right)$ with the rotation phase. A possible anti-correlation between the $\mathrm{BD}$ and radar albedo is observed. The mass-deficit region, characterized as having the highest radar albedo, also shows the highest value for the spectral slope and the minimum BD, while the antipode of this region, where the radar albedo reaches its lowest value, shows the maximum BD and less steep spectral slopes.

Band center values were used to calculate the pyroxene chemistry of Psyche, which ranges from $\mathrm{Fs}_{26.7 \pm 3.3} \mathrm{Wo}_{3.5 \pm 1.1}$ to $\mathrm{Fs}_{48.2 \pm 3.3} \mathrm{Wo}_{11.8 \pm 1.1}$. The lowest value of Fs is found for bodycentered longitudes of $41^{\circ}$ and $309^{\circ}$, corresponding to possible excavated regions, including a crater-like depression $85 \pm 20 \mathrm{~km}$ in diameter located at a longitude of $300^{\circ}$. The highest values of Fs could be the result of an overestimation caused by the presence of a secondary or accessory mineral (e.g., olivine or high-Ca pyroxene). We determined that an average value of $\mathrm{Fs}_{30} \mathrm{En}_{65} \mathrm{Wo}_{5}$ is likely more representative of the pyroxene chemistry of Psyche.

Using a new laboratory spectral calibration method, we estimated an average pyroxene abundance of $6 \% \pm 1 \%$. The spectral characteristics of Psyche on a global scale suggest that the metal on the surface and the silicate component are well mixed, as indicated by the presence in all of the spectra of a weak pyroxene absorption band in the $0.9 \mu \mathrm{m}$ region. Furthermore, the distribution of a secondary phase, possibly high-Ca pyroxene, across the surface of Psyche suggests that this is an indigenous mineral derived from the parent body.

The apparent lack of olivine on the surface of Psyche could indicate that pyroxene and not olivine was the first crystallizing mineral in the mantle of the parent body. Another possible explanation could involve the disruption and reaccretion of a differentiated asteroid with a molten core, followed by subsequent hit-and-run collisions of the reaccreted body. In this scenario, the olivine fraction is reduced during the reaccretion process, and the hit-and-run collisions leave behind an exposed metallic core covered by a regolith layer of metal and pyroxene.

The effects of the phase angle, grain size, and space weathering on the spectral data were examined. We observed that these factors could be responsible to some extent for the variations seen in the band parameters. However, since they are difficult to disentangle, it is not possible to know how significant their contributions are.

This research work was supported by NASA Planetary Mission Data Analysis Program Grant NNX13AP27G, NASA NEOO Program Grant NNX12AG12G, and NASA Planetary Geology and Geophysics Program Grant NNX11AN84G. We thank the IRTF TAC for awarding time to this project, and we thank the IRTF TOs and MKSS staff for their support. The IRTF is operated by the University of Hawaii under Cooperative Agreement no. NCC 5-538 with the National Aeronautics and Space Administration, Office of Space Science, Planetary Astronomy Program. Part of this work was done at the Arecibo Observatory, which is operated by SRI International under a cooperative agreement with the National Science Foundation (AST-1100968) and in alliance with Ana G. Mendez-Universidad Metropolitana and the Universities Space Research Association. The Arecibo Planetary Radar Program is supported by the National Aeronautics and Space Administration under Grant Nos. NNX12AF24G and NNX13AQ46G, issued through the Near Earth Object Observations program. E.A.C. thanks the Canada Foundation for Innovation, the Manitoba Research Innovations Fund, the 
Canadian Space Agency, the Natural Sciences and Engineering Research Council of Canada, and the University of Winnipeg for supporting the establishment and ongoing operation of the University of Winnipeg's Planetary Spectrophotometer Facility. The authors would like to thank the anonymous reviewer for their comments, which helped improve the manuscript. We also thank Kim Tait and Ian Nicklin from the Royal Ontario Museum for providing a suite of mesosiderite samples for this study.

\section{REFERENCES}

Asphaug, E., Agnor, C. B., \& Williams, Q. 2006, Natur, 439, 155 Asphaug, E., \& Reufer, A. 2014, NatGe, 7, 564

Bell, J. F., Davis, D. R., Hartmann, W. K., \& Gaffey, M. J. 1989, in Asteroids II, Asteroids - The big picture (Tucson, AZ: Univ. Arizona Press), 921

Binzel, R. P., Bus, S. J., Xu, S., et al. 1995, Icar, 117, 443

Boesenberg, J. S., Delaney, J. S., \& Prinz, M. 1997, in 28th Annual Lunar and Planetary Science Conf., Magnesian megacrysts and matrix in the mesosiderite Lamont (Hampton, VA: STI), 125

Brunetto, R., Vernazza, P., Marchi, S., et al. 2006, Icar, 184, 327

Burbine, T. H., Buchanan, P. C., \& Binzel, R. P. 2007, in 38th Lunar and Planetary Science Conf., Deriving Formulas from HED Spectra for Determining the Pyroxene Mineralogy of Vesta and Vestoids, 2117

Chapman, C. R., \& Gaffey, M. J. 1979, in Asteroids, Reflectance spectra for 277 asteroids, ed. T. Gehrels (Tucson, AZ: Univ. Arizona Press), 655

Chapman, C. R., \& Salisbury, J. W. 1973, Icar, 19, 507

Clark, B. E., Bus, S. J., Rivkin, A. S., et al. 2004, AJ, 128, 3070

Clark, R. N., \& Roush, T. L. 1984, JGR, 89, 6329

Cloutis, E. A., Gaffey, M. J., Jackowski, T. L., et al. 1986, JGR, 91, 11641

Cloutis, E. A., Hardersen, P. S., Bish, D. L., et al. 2010, M\&PS, 45, 304

Cloutis, E. A., Hardersen, P. S., Reddy, V., et al. 2009, in 40th Lunar and Planetary Science Conf., Metal-Orthopyroxene and Metal-Olivine Mixtures: Spectral Reflectance Properties and Implications for Asteroid Spectroscopy, 1332

Cloutis, E. A., Sanchez, J. A., Reddy, V., et al. 2015, Icar, 252, 39

Cushing, M. C., Vacca, W. D., \& Rayner, J. T. 2004, PASP, 116, 362

Elkins-Tanton, L. T., Asphaug, E., Bell, J., et al. 2014, in 45th Lunar and Planetary Science Conf., Journey to a Metal World: Concept for a Discovery Mission to Psyche, 1253
Elkins-Tanton, L. T., Asphaug, E., Bell, J., et al. 2015, in 46th Lunar and Planetary Science Conf., The Discovery Science of Asteroid (16) Psyche, 1632

Elkins-Tanton, L. T., Weiss, B. P., Asphaug, E., et al. 2013, in 44th Lunar and Planetary Science Conf., Differentiation in Planetesimals with Applications to Asteroid (16) Psyche, 1351

Fornasier, S., Clark, B. E., Dotto, E., et al. 2010, Icar, 210, 655

Gaffey, M. J. 2010, Icar, 209, 564

Gaffey, M. J., Burbine, T. H., Piatek, J. L., et al. 1993, Icar, 106, 573

Gaffey, M. J., Cloutis, E. A., Kelley, M. S., et al. 2002, in Asteroids III, Mineralogy of Asteroids, ed. W. F. Bottke, Jr. et al. (Tucson, AZ: Univ. Arizona Press), 183

Hapke, B. 2001, JGR, 106, 10039

Hardersen, P. S., Cloutis, E. A., Reddy, V., et al. 2011, M\&PS, 46, 1910

Hardersen, P. S., Gaffey, M. J., \& Abell, P. A. 2005, Icar, 175, 141

Kaasalainen, M., Torppa, J., \& Piironen, J. 2002, Icar, 159, 369

Kuzmanoski, M., \& Kovačević, A. 2002, A\&A, 395, L17

Magri, C., Ostro, S. J., Scheeres, D. J., et al. 2007, Icar, 186, 152

Matter, A., Delbo, M., Carry, B., et al. 2013, Icar, 226, 419

Mittlefehldt, D. W., McCoy, T. J., Goodrich, C. A., et al. 1998, in Planetary Materials, Vol. 36, ed. J. J. Papike

Neeley, J. R., Clark, B. E., Ockert-Bell, M. E., et al. 2014, Icar, 238, 37

Ockert-Bell, M. E., Clark, B. E., Shepard, M. K., et al. 2008, Icar, 195, 206

Ockert-Bell, M. E., Clark, B. E., Shepard, M. K., et al. 2010, Icar, 210, 674

Ostro, S. J., Campbell, D. B., \& Shapiro, I. I. 1985, Sci, 229, 442

Pieters, C. M., Taylor, L. A., Noble, S. K., et al. 2000, M\&PS, 35, 1101

Rayner, J. T., Toomey, D. W., Onaka, P. M., et al. 2003, PASP, 115, 362

Reddy, V., Le Corre, L., O'Brien, D. P., et al. 2012, Icar, 221, 544

Sanchez, J. A., Michelsen, R., Reddy, V., \& Nathues, A. 2013, Icar, 225, 131

Sanchez, J. A., Reddy, V., Dykhuis, M., et al. 2015, ApJ, 808, 93

Sanchez, J. A., Reddy, V., Nathues, A., et al. 2012, Icar, 220, 36

Scott, E. R. D., Haack, H., \& Love, S. G. 2001, M\&PS, 36, 869

Shepard, M. K., Clark, B. E., Nolan, M. C., et al. 2008, Icar, 195, 184

Shepard, M. K., Richardson, J., Taylor, P. A., et al. 2017, Icar, 281, 388

Takir, D., Reddy, V., Sanchez, J., \& Shepard, M. K. 2016, in AAS/Division for Planetary Sciences Meeting 48 Abstracts, \#510.01

Tedesco, E. F., Noah, P. V., Noah, M., \& Price, S. D. 2002, AJ, 123, 1056

Tholen, D. J. 1984, PhDT, Univ. Arizona

Weisberg, M. K., McCoy, T. J., \& Krot, A. N. 2006, in Meteorites and the Early Solar System II, Systematics and Evaluation of Meteorite Classification, ed. S. Lauretta \& H. Y. McSween, Jr. (Tucson, AZ: Univ. Arizona Press), 19

Zellner, B., \& Gradie, J. 1976, AJ, 81, 262 